\title{
Analytical Reformulation of Security Constrained Optimal Power Flow with Probabilistic Constraints
}

\section{Conference Paper}

Author(s):

Roald, Line; Oldewurtel, Frauke; Krause, Thilo; Andersson, Göran

Publication date:

2013

Permanent link:

https://doi.org/10.3929/ethz-a-010607821

Rights / license:

In Copyright - Non-Commercial Use Permitted

Originally published in:

https://doi.org/10.1109/PTC.2013.6652224 


\title{
Analytical Reformulation of Security Constrained Optimal Power Flow with Probabilistic Constraints
}

\author{
Line Roald, Student Member, IEEE, Frauke Oldewurtel, Member, IEEE, \\ Thilo Krause, Member, IEEE, Göran Andersson, Fellow, IEEE \\ Power Systems Laboratory \\ Department of Electrical Engineering \\ ETH Zurich, Switzerland \\ Email: $\{$ roald | oldewurtel | krause | andersson\}@eeh.ee.ethz.ch
}

\begin{abstract}
In this paper, uncertainties from wind power in-feed are taken into account in a DC security-constrained optimal power flow (SCOPF) by formulating probabilistic constraints. The deviations from the wind power forecast are represented as Gaussian random variables and an analytical reformulation of the constraints is proposed, which is exact for the Gaussian distribution. The resulting formulation has the same computational complexity as the deterministic problem. Furthermore, a valuation framework to assess the cost of securing the system against fluctuations in wind power in-feed is proposed. The applicability of the method and the valuation framework is demonstrated on the IEEE RTS96 system. We show that the probabilistic formulation leads to lower probability of thermal overloads, and that it is less costly to secure the system against uncertain in-feed than to secure the system against failures in most cases.
\end{abstract}

\section{INTRODUCTION}

Increasing penetration of fluctuating renewable energy sources (RES) and the liberalization of energy markets lead to higher uncertainties in transmission network operation [1]. Traditionally, the deterministic N-1 security criterion has been used to assess power system security. However, several incidents over the last decade have indicated that this assessment might no longer be sufficient as a guarantee against blackouts [2]. In [2], it is argued that additional information is needed to operate the system securely while maintaining a reasonably low cost, and that this additional information could be provided in terms of a so-called risk index. Here, we propose a novel probabilistic approach to include information on uncertainties originating from RES in-feed into day-ahead operational planning.

Optimal power flow (OPF) is a fundamental tool for static power system network calculations [3]. To find an N-1 secure dispatch, additional constraints can be added to the OPF to consider the effect of component failures, leading to a security constrained optimal power flow (SCOPF). In [4], a SCOPF is formulated using a DC power flow which allows the incorporation of security constraints based on linear sensitivity factors. OPF and SCOPF are deterministic problems.

To incorporate Gaussian uncertainty of load in the power flow equations, the probabilistic load flow method was proposed in the 70s [5]. It has been extended to account for other sources of uncertainty with different probability distributions, such as wind power in-feed [6] or branch outages [7]. In [8], a probabilistic load flow was combined with an OPF to get a probabilistic optimal power flow (pOPF). A natural extension is to introduce probabilistic constraints in the SCOPF to form a probabilistic security constrained optimal power flow (pSCOPF), as is proposed in this paper.

A similar approach is presented in [9], where a DC pSCOPF is solved to find a dispatch that guarantees $\mathrm{N}-1$ security for the next day with a given probability. The probabilistic constraints are reformulated using a sampling based approach. In contrast, we propose to use an analytic reformulation of the probabilistic constraints, which is exact for the Gaussian distribution.

The contribution of this paper is threefold. First, the methods of probabilistic load flow and DC SCOPF are combined and the probabilistic constraints reformulated analytically, as presented in Section II. Second, a valuation framework to explicitly estimate the cost of securing the system against failures and uncertainty in RES in-feed is proposed (Section III). Finally, solutions obtained with the four different optimization formulations, i.e. OPF, pOPF, SCOPF and pSCOPF, are compared for a test network using the valuation framework, as presented in Section IV. Section V summarizes and concludes.

\section{Problem Formulation}

The problem formulation is presented in two steps. First, considerations regarding the modeling of wind power and regulating power are presented. In the second part, the four formulations are derived, starting with a standard DC OPF which is successively extended with appropriate constraints to incorporate the above mentioned security and uncertainty considerations.

\section{A. Wind Power Modelling}

Wind power in-feed is modeled as the sum of forecast and forecast error

$$
P_{W}=P_{W}^{f c}+\Delta P_{W} .
$$

$P_{W}$ is the actual wind power in-feed, $P_{W}^{f c}$ corresponds to the forecasted values and $\Delta P_{W}$ is the deviation from the forecast. Using the same reasoning as in [10], the forecast errors $\Delta P_{W}$ are represented as Gaussian random variables with zero mean. The standard deviations $\sigma_{w}$ of each of the 
wind farms are related to the installed capacity at the respective bus, as proposed in [11]. The forecast errors are assumed to be spatially uncorrelated.

\section{B. Sensitivity Factors and Regulating Power}

In the following, a system with $N_{B}$ buses, $N_{L}$ lines, $N_{G}$ conventional generators, and $N_{W}$ buses with wind power infeed is considered. The vector $P_{G}$ describes the active power injections of generators, with $P_{G}^{\max }$ being the upper nominal production level. The active power consumption of the loads is given by $P_{L}$, and $\theta$ is the vector of voltage angles at each bus. Three types of sensitivity factors are used to compute changes in line flows and generation, which are similar to the factors used in [4], [12] and [9]:

- Line Outage Distribution Factors (LFs) for line flow changes due to line outages.

- Generalized Generation Distribution Factors (GFs) for line flow changes due to deviations from generation schedule.

- A weighting matrix $(d)$ for generation changes due to deviations from generation schedule.

The LFs describe the influence of each line outage on all other lines, thus forming a $N_{L} \times N_{L}$ matrix, with diagonal elements equal to 0 . The fraction of the power flow on line $m n$ that is shifted to line $i j$ in case of an outage of line $m n$ is given by [4]:

$$
L F_{i j, m n}=\frac{x_{m n}}{x_{i j}} \cdot \frac{X_{i m}-X_{i n}-X_{j m}+X_{j n}}{x_{m n}-\left(X_{m m}+X_{n n}-2 X_{m n}\right)} .
$$

Here, $x_{i j}$ is the line reactance of line $i j$, and $X_{i m}$ refers to the the entry at the $i$ th row and $m$ th column of the bus reactance matrix.

In the computation of GFs and $d$, the power needed to compensate deviations in generation is distributed among all conventional generators based on their nominal power. GF is a $N_{L} \times\left(N_{G}+N_{W}\right)$ matrix, and the entry describing the change in line flow on line $i j$ after outage of generation unit $k$ is given by

$$
G F_{i j}^{k}=\frac{1}{x_{i j}} \mathbf{1}_{i j}^{T} \mathbf{X} \mathbf{r}_{k}
$$

where $\mathbf{1}_{i j}=\mathbf{1}_{i}-\mathbf{1}_{j}$ and $\mathbf{1}_{i}$ is a vector with the $i$ th entry is 1 and all other elements are $0 . \mathbf{r}_{k}$ is a vector describing how a decrease in generation at node $k$ is compensated by increased generation at the other nodes. The $k$ th entry, corresponding to the $k$ th node, is given by

$$
\mathbf{r}_{k}(k)=\frac{\sum_{g \in \text { node } k, g \neq k} P_{G, g}^{\max }}{\sum_{j=1, j \neq k}^{N_{G}} P_{G, j}^{\max }}-1,
$$

where $P_{G, g}^{\max }$ is the maximum nominal generation of generator $g$. The entries for all other nodes are given by

$$
\mathbf{r}_{k}(i)=\frac{\sum_{g \in \text { node } i} P_{G, g}^{\max }}{\sum_{j=1, j \neq k}^{N_{G}} P_{G, j}^{\max }} .
$$

The element $d_{g, k}$ of the weighting matrix describes the fraction of the missing production at generator $k$ that is compensated by the conventional generator $g$ :

$$
d_{g, k}=\frac{P_{G, g}^{\max }}{\sum_{j=1, j \neq k}^{N_{G}} P_{G, j}^{\max }} .
$$

If $k$ is a wind farm, the denominator includes contributions from all conventional generators. In total, the weighting matrix has $N_{G} \times\left(N_{G}+N_{W}\right)$ entries.

\section{Standard Optimal Power Flow}

Including wind power generation, the formulation for the standard DC optimal power flow is

$$
\min C_{G}^{T} P_{G}
$$

subject to

$$
\begin{aligned}
P_{G}+P_{W}-B \theta & =P_{L} \\
P_{W} & =P_{W}^{f c} \\
P_{G}^{\min } \leq P_{G} & \leq P_{G}^{\max } \\
-P_{i j}^{\max } \leq \frac{1}{x_{i j}}\left(\theta_{i}-\theta_{j}\right) & \leq P_{i j}^{\max } \quad \text { for all lines } i j \\
\theta_{\text {slack }} & =0
\end{aligned}
$$

The objective is to minimize generation cost, as described by (7). The DC power flow equations are given by (8), with $B$ being the bus admittance matrix. As the wind power production is non-dispatchable, the wind power in-feed is set equal to the forecasted values in (9). Eq. (10) constrains generation to be within the minimum and maximum levels $P_{G}^{\min }$ and $P_{G}^{\max }$. Constraints on the line flows are given by (11), and the angle of the slack bus is set to zero by (12).

\section{Probabilistic Optimal Power Flow}

Random variations in wind power in-feed lead to changes in the line flows. The line constraint (11), considering only the upper bound, becomes

$$
\frac{1}{x_{i j}}\left(\theta_{i}-\theta_{j}\right)+\sum_{w=1}^{N_{W}} G F_{i j, w} \Delta P_{W, w} \leq P_{i j}^{\max } .
$$

Here, $\Delta P_{W, w}$ is the variation in wind power in-feed from wind farm $w . G F_{i j, w}$ describes how the variations in power in-feed from wind farm $w$ influences line $i j$, as is seen from the term $\sum_{w=1}^{N_{W}} G F_{i j, w} \Delta P_{W, w}$. Due to the random nature of $\Delta P_{W}$, this constraint is not tractable. To create a tractable constraint, Eq. (13) is first formulated as a probabilistic constraint, which requires a condition to be met with a predefined probability. The probabilistic formulation of the line constraint (11), including random wind power in-feed and considering only the upper bound, is thus given by

$\mathbb{P}\left[\frac{1}{x_{i j}}\left(\theta_{i}-\theta_{j}\right)+\sum_{w=1}^{N_{W}} G F_{i j, w} \Delta P_{W, w}-P_{i j}^{\max } \leq 0\right] \geq 1-\varepsilon$ 
where $\varepsilon$ denotes the violation probability. Using the properties of the Gaussian distribution, this constraint can be reformulated analytically to the following tractable constraint [13]:

$$
\frac{1}{x_{i j}}\left(\theta_{i}-\theta_{j}\right) \leq P_{i j}^{\max }-\Phi^{-1}(1-\varepsilon) \sqrt{\sum_{w=1}^{N_{W}} G F_{i j, w}^{2} \sigma_{w}^{2}} .
$$

Accounting for wind power uncertainty leads to a tightening of the constraint, as observed when comparing (15) with (11). The tightening increases with increasing uncertainty $\left(\sigma_{w}\right.$ larger) and decreasing violation probability ( $\varepsilon$ smaller).

A probabilistic constraint for the generation capacity, again considering only the upper bound, is given by

$$
\mathbb{P}\left[P_{G, g}+\sum_{w=1}^{W} d_{g, w} \Delta P_{W, w} \leq P_{G, g}^{\max }\right] \geq 1-\varepsilon,
$$

where $d_{g, w}$ is the part of $\Delta P_{W, w}$ that must be compensated by generator $g$. Using the same analytic reformulation as above yields

$$
P_{G, g} \leq P_{G, g}^{\max }-\Phi^{-1}(1-\varepsilon) \sqrt{\sum_{w=1}^{W} d_{g, w}^{2} \sigma_{w}^{2}}
$$

Again, the presence of uncertainty leads to a tighter constraint, as seen when comparing (17) to (10). The generation is constrained so as to ensure enough reserve for compensation of wind power fluctuations.

\section{E. Security-Constrained Optimal Power Flow}

A SCOPF includes constraints to guarantee N-1 security. These constraints are formulated using linear sensitivity factors, which are exact for a DC power flow. The contingency set contains outages of any generator or line, and considers only preventive action. The security constraints for line $i j$ are

$$
\begin{aligned}
\frac{1}{x_{i j}}\left(\theta_{i}-\theta_{j}\right)+L F_{i j, m n} \frac{1}{x_{m n}}\left(\theta_{m}-\theta_{n}\right) & \leq \alpha \cdot P_{i j}^{\max } \\
\frac{1}{x_{i j}}\left(\theta_{i}-\theta_{j}\right)+G F_{i j, g} P_{G, g} & \leq \alpha \cdot P_{i j}^{\max } .
\end{aligned}
$$

The flow on line $i j$ after the outage of line $m n$ is constrained by (18). The flow on line $i j$ after outage of generation unit $P_{G, g}$, which can be either a conventional generator or a wind farm, is constrained by (19). The factor $\alpha \geq 1$ is added to allow for an emergency line flow that is higher than the steady state limit. For the generators, additional constraints are added to account for generation outages. Generation at generator $g$ after outage of generator $k$ is constrained by

$$
P_{G, g}+d_{g, k} P_{G, k} \leq \beta \cdot P_{G, g}^{\max },
$$

where $d_{g, k}$ is the part of the outaged generation $P_{G, k}$ that must be compensated by generator $g$. The factor $\beta \geq 1$ is added to allow for increased loading of the generators (i.e. use of regulating power) in case of a failure.

\section{F. Probabilistic Optimal Power Flow with Security Con- straints}

The security constraints of the SCOPF can also be reformulated to account for random wind power in-feed. The line constraint on line $i j$ due to outage of line $m n$ (18) is given as

$$
\begin{gathered}
\frac{1}{x_{i j}}\left(\theta_{i}-\theta_{j}\right)+L F_{i j, m n} \frac{1}{x_{m n}}\left(\theta_{m}-\theta_{n}\right) \leq \alpha \cdot P_{i j}^{\max }- \\
\Phi^{-1}(1-\varepsilon) \sqrt{\sum_{w=1}^{N_{W}}\left(G F_{i j, w}^{2}+L F_{i j, m n}^{2} G F_{m n, w}^{2}\right) \sigma_{W, w}^{2}} .
\end{gathered}
$$

For constraints considering generation outages, one needs to distinguish the outage of a wind farm (which leads to reduced uncertainty) and the outage of a conventional generator. The probabilistic constraints on line $i j$ due to generation outages are given by

$$
\begin{aligned}
& \frac{1}{x_{i j}}\left(\theta_{i}-\theta_{j}\right)+G F_{i j, v} P_{W, w}^{f c} \leq \alpha \cdot P_{i j}^{\max }- \\
& \Phi^{-1}(1-\varepsilon) \sqrt{\sum_{w=1, w \neq v}^{N_{W}} G F_{i j, w}^{2} \sigma_{w, v}^{2}}
\end{aligned}
$$

for the outage of a wind farm $v$ and by

$$
\begin{aligned}
& \frac{1}{x_{i j}}\left(\theta_{i}-\theta_{j}\right)+G F_{i j, k} P_{G, k} \leq \alpha \cdot P_{i j}^{\max }- \\
& \Phi^{-1}(1-\varepsilon) \sqrt{\sum_{w=1}^{N_{W}}\left(G F_{i j, w}^{2}+G F_{i j, k}^{2} d_{k}^{2}\right) \sigma_{w, v}^{2}}
\end{aligned}
$$

for the outage of a conventional generator $k$. Similarly, the constraints on generator $g$ due to the outage of the wind farm $v$ can be formulated as

$$
\begin{aligned}
& P_{G, g}+d_{g, v} P_{W, v}^{f c} \leq \beta \cdot P_{G, g}^{\max }- \\
& \Phi^{-1}(1-\varepsilon) \sqrt{\sum_{w=1, w \neq v}^{N_{W}} d_{g, w}^{2} \sigma_{w}^{2}}
\end{aligned}
$$

and the constraint due to the outage of generator $k$ as

$$
\begin{aligned}
P_{G, g}+d_{g, k} P_{G, k} \leq \beta \cdot P_{G, g}^{\max }- & \\
& \Phi^{-1}(1-\varepsilon) \sqrt{\sum_{w=1}^{N_{W}}\left(d_{g, w}^{2}+d_{g, k}^{2} d_{k, w}^{2}\right) \sigma_{w}^{2}} .
\end{aligned}
$$

Comparing the probabilistic security constraints in the pSCOPF (e.g. (23) and (24)) with the deterministic security constraints of the SCOPF (e.g. (19)) shows that accounting for uncertainty leads to tighter constraints.

A main advantage of the proposed formulation is that the probabilistic problem has the same computational complexity as the deterministic problem. The problem remains linear with the same number of constraints as the deterministic problem, and is thus efficiently solvable by standard software. 
TABLE I

GENERATOR DATA

\begin{tabular}{|c|c|c|c|}
\hline Bus & Generator \# & Cost [\$/p.u.h.] & $P_{G}^{\max }$ \\
\hline 1,2 & $1,2,5,6$ & 24.842 & 20 \\
1,2 & $3,4,7,8$ & 10.239 & 76 \\
7 & $9-11$ & 17.974 & 100 \\
13 & $12-14$ & 18.470 & 197 \\
15 & $15-19$ & 21.227 & 12 \\
$15,16,23$ & $20,21,30,31$ & 9.537 & 155 \\
18,21 & 22,23 & 5.230 & 400 \\
22 & $24-29$ & 1 & 50 \\
23 & 32 & 9.587 & 350 \\
\hline
\end{tabular}

\section{VALUATION FRAMEWORK}

The solution obtained with an OPF can be seen as the most desirable generation dispatch from a regulation authority point of view, as it ensures efficient operation of both generation units and the transmission grid [12]. Incorporating additional constraints to account for security and uncertainty considerations not only leads to increased security but also to higher generation cost. Solving all four optimization problems allows for explicit analysis of the trade-off between operational security and cost. We propose to use two measures:

- Cost of Security $(\mathrm{CoS})$ : Cost difference between the OPF and the SCOPF (as defined in [12])

$$
\text { CoS }=\text { Cost }_{S C O P F}-\text { Cost }_{O P F} .
$$

- Cost of Uncertatinty (CoU): Cost difference between a deterministic and a probabilistic OPF

$$
\begin{aligned}
& \operatorname{CoU}_{O P F}=\text { Cost }_{\text {POPF }}-\text { Cost }_{O P F}, \\
& \operatorname{CoU}_{S C O P F}=\text { Cost }_{p S O P F}-\text { Cost }_{S C O P F} .
\end{aligned}
$$

When referring to both of these measures, the term $\mathrm{CoU}$ without subscript is used.

While CoS estimates the cost of enforcing the $\mathrm{N}-1$ criterion, $\mathrm{CoU}$ gives an impression of the system cost related to integration of renewable energy.

\section{CAse Study}

The influence of system uncertainty on total generation cost is studied based on the 24-bus system shown in Fig. 1. The system corresponds to the IEEE One Area RTS-96 system, as described in [14], with some modifications as follows. The line capacities are reduced to $80 \%$ of the steady state capacity listed in [14], since only active power flows are considered. Aggregations of wind power in-feed are located at bus 8 and bus 15, with an installed capacity of 500 and $700 \mathrm{MW}$, respectively. The generation costs used in the OPF are the linear cost components from [15], and are listed with other generator data in Table I. We consider a peak load situation, where the total system load equals $2850 \mathrm{MW}$ and the total dispatchable generation capacity (not including wind energy) is $3405 \mathrm{MW}$.

In the base case scenario, the forecasted wind power in-feed equals $25 \%$ of installed wind power capacity, with $125 \mathrm{MW}$ in-feed at bus 8 and $175 \mathrm{MW}$ in-feed at bus 15. Based on

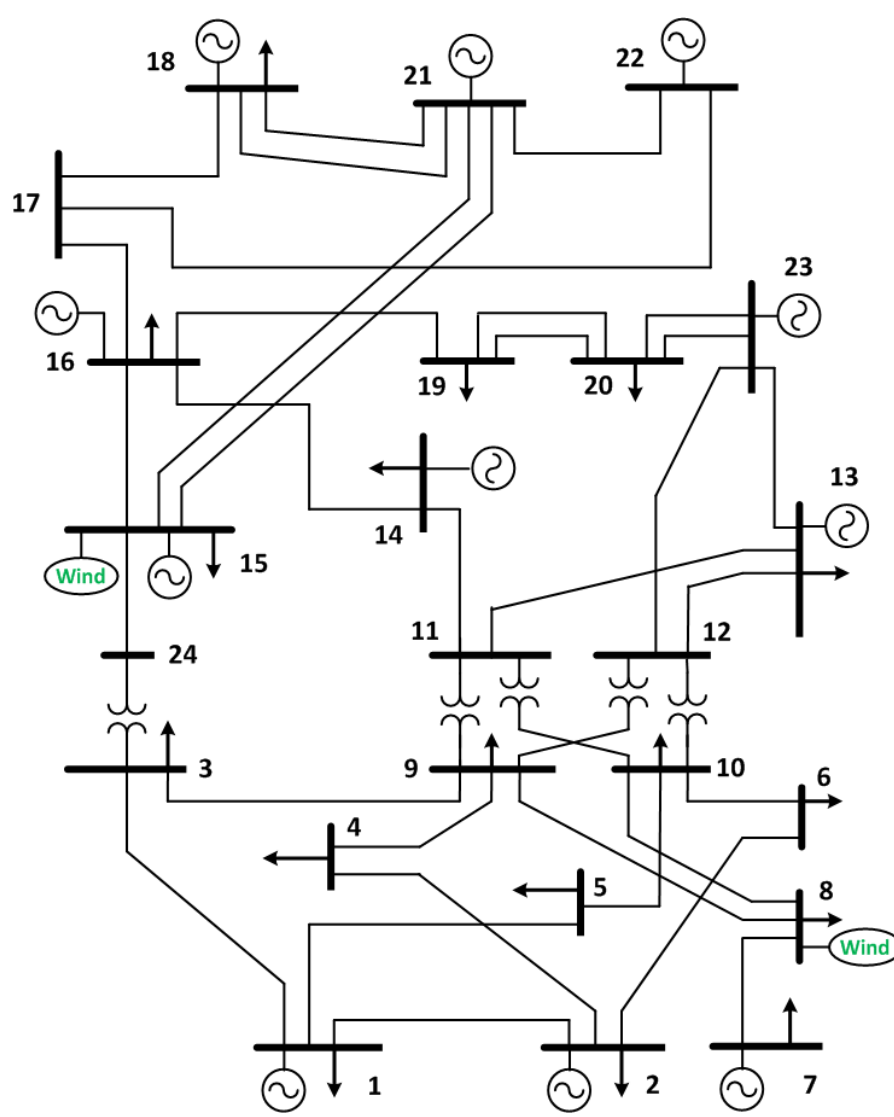

Fig. 1. 24-bus test system with wind power in-feed at bus 8 and bus 15 .

results from [11], the standard deviation $\sigma_{W}$ is assumed to be $7.5 \%$ of the installed capacity. The violation probability $\varepsilon$ is chosen to be $5 \%$. The emergency line limit is chosen to be $10 \%$ higher than the steady state limit, i.e. $\alpha=1.1$. Each generator is assumed to have $25 \%$ of their nominal capacity available as regulating power in case of a contingency, i.e. $\beta=1.25$.

\section{A. Investigations}

The following cases are investigated:

1) Constraint tightening: An example of how the constraint tightening reduces violation probability is shown to illustrate the purpose of the probabilistic method. Further, as the influence of the wind power fluctuations is not the same throughout the system, the influence on different lines is investigated. This is done by calculating the constraint tightening, i.e. reduction in available line capacity due to wind power uncertainty, for each of the lines and compare them to each other.

2) Effect of uncertainty on cost: The generation cost of the optimal dispatch from each of the four different optimization problems is calculated for the base case described above. The results give an impression of $\mathrm{CoS}$ and $\mathrm{CoU}$ for the base case.

3) Sensitivity with regard to load level: The base case considers a load level corresponding to the peak system load. To find out how $C o S$ and $C o U$ depend on the load level, generation cost is calculated for a range of different load levels, 


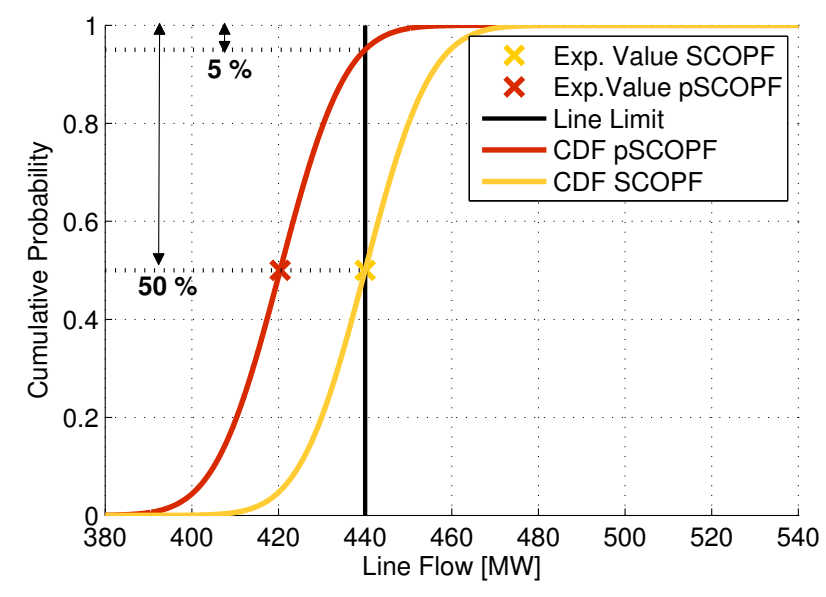

Fig. 2. Expected value (EV) of the power flowing on line 23 (bus 14-16) after outage of line 7 (bus 2-3), as computed with SCOPF and pSCOPF. Superimposed on the EV is the cumulative distribution function (CDF) of the line flow. Because of the constraint tightening in the pSCOPF, the probability of violating the line limit constraint is reduced from $50 \%$ to $5 \%$.

$P_{L}=\frac{X \%}{100} \cdot P_{L, p e a k}$ with $X=\{20,40,60,80,100\}$.

4) Sensitivity with regard to forecast uncertainty: To assess how the level of forecast uncertainty influences $C o U$, generation cost is computed for different $\sigma_{W}$.

\section{B. Results}

1) Constraint tightening: Fig. 2 shows the expected values for line flow on line 23 (bus 14-16) after the outage of line 7 (bus 3-24) as obtained with SCOPF and pSCOPF, with the cumulative distribution function of the line flow superimposed. Due to the constraint tightening in the pSCOPF case, the expected line flow is shifted from $440 \mathrm{MW}$ to $420 \mathrm{MW}$. This leads to a reduction of the probability of constraint violation from $50 \%$ with the SCOPF to $5 \%$ with the pSCOPF, and thus decreases the probability of cascading overloads.

The tightening of the line capacity limits is shown in Fig. 3 . The largest constraint tightening occurs for lines that are related to power transfer from the buses where wind energy is produced (i.e. lines 12, 13 and 24), for some of the lines that transport power from the generators in the north to the loads in the south (i.e. lines 19 and 23 through bus 14), as well as for lines connected to large generators taking a major share in balancing the wind power deviations (i.e. line 29 between bus 18 and 17). These are the lines that are most influenced by deviations in the wind power in-feed.

2) Effect of uncertainty on cost: In Fig. 4, generation cost of the optimal dispatch from each of the four different optimization problems is shown for the base case described above. The difference in cost between the OPF and the SCOPF is approximately twice as high as the difference between the deterministic and probabilistic solutions, $\mathrm{CoS} \approx 2 \cdot \mathrm{CoU}$. The cost of imposing security constraints on the system, i.e. adding new constraints, is thus higher than the cost induced by constraint tightening due to uncertainty.

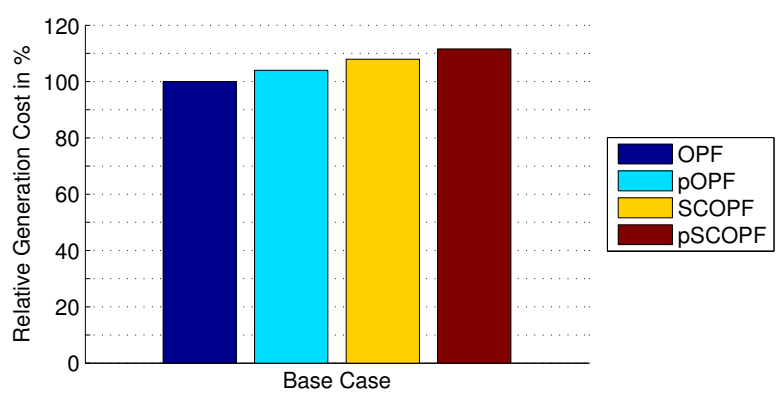

Fig. 4. Total generation cost of the optimal dispatch computed with OPF, pOPF, SCOPF and pSCOPF.

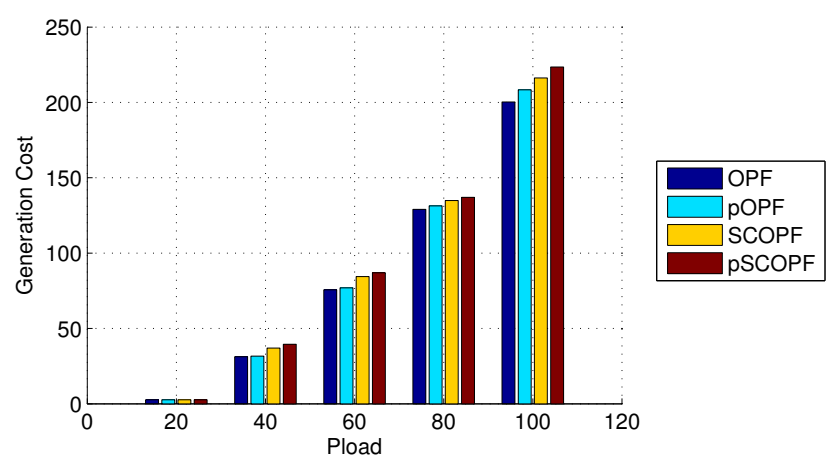

Fig. 5. Total (absolute) generation cost of optimal dispatch for different load levels. At lower load levels, $\mathrm{CoS}>\mathrm{CoU}$.

3) Sensitivity with regard to load level: The results obtained for different load levels are shown in Fig. 5. At very low load levels $(20 \%)$, the consideration of uncertainty and security constraints does not add any cost such that $C o S=C o U=0$. In such low load situations, there is enough transfer capacity available to produce power at the cheapest power plants, even when security constraints and tightening due to uncertainty is introduced. At intermediate load levels (40-60\%), the difference between the $C o S$ and $C o U$ is larger than in the peak load case, with $C o S>4 \cdot C o U$. In high load situations (80-100\%), the $C o S \approx 2 \cdot C o U$. This means that the relative magnitude of $C o U$ compared with $C o S$ is larger in high load situation than in situations where the system is under less pressure. In a situation where the flows through the system are already high, decreasing the transfer capacity has a higher impact on the generation cost.

4) Sensitivity with regard to forecast uncertainty: In Fig. 6, the generation cost is shown for different values of $\sigma_{W}$. The cost increase is linearly related to the increase of the standard deviation up to a standard deviation of $12.5 \%$. With higher standard deviations, the cost of the pSCOPF dispatch increase more rapidly. This is because additional and more expensive generators (i.e. generators at bus 1 and 2) are dispatched to ensure feasibility. At the highest considered uncertainty level with $\sigma_{W}=15 \%$, the influence from the random variations is so high that $\operatorname{CoS}<\mathrm{CoU}$. The uncertainty has reached a level 


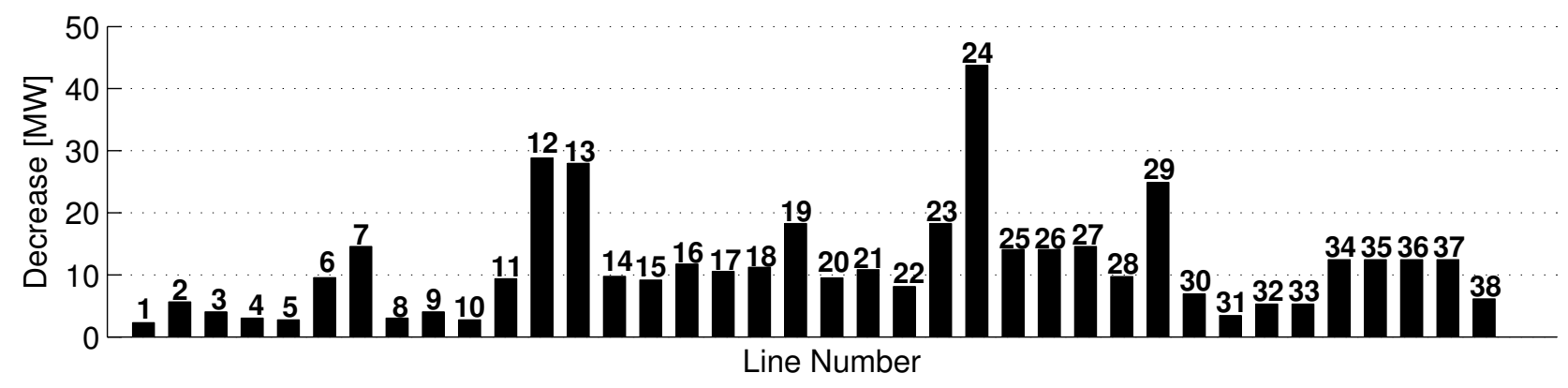

Fig. 3. Decrease of available capacity on the lines due to uncertainty.

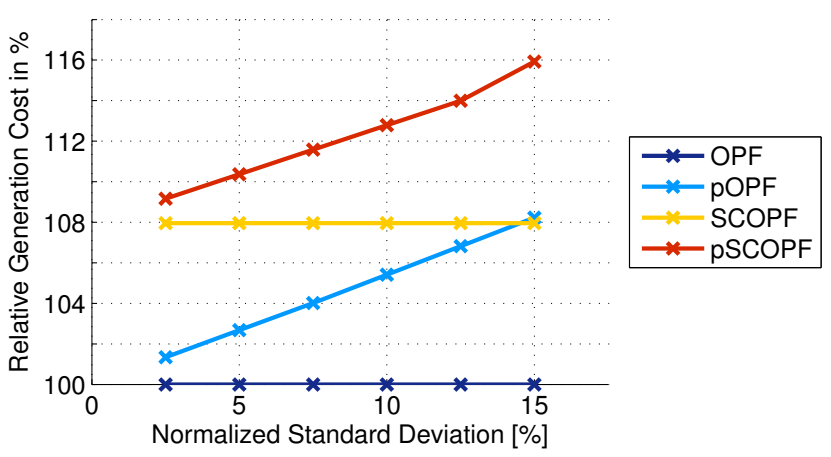

Fig. 6. Total generation cost of optimal dispatch for different values of $\sigma_{W}$.

where the constraint tightening increases the cost more than the additional security constraints.

\section{CONCLUSION}

In this paper, a security constrained DC optimal power flow with probabilistic constraints (pSCOPF) was formulated based on linear sensitivity factors and Gaussian uncertainty. The approach assumes that the random fluctuations in wind energy infeed can be represented as Gaussian random variables. Using the properties of the Gaussian distribution, the probabilistic constraints were reformulated analytically. The resulting probabilistic OPF problem has the same computational complexity as the deterministic OPF problem, as it remains linear and has the same number of constraints.

A valuation framework to estimate cost related to secure grid integration of renewables, so called Cost of Uncertainty (CoU), was proposed. It was used together with the measure Cost of Security ( $\mathrm{CoS}$ ) from [12], which estimates the cost of ensuring $\mathrm{N}-1$ security, to evaluate the relative costs to secure the system against uncertainties from RES and contingencies.

$\mathrm{CoU}$ and $\mathrm{CoS}$ were computed for a modified version of the IEEE One Area RTS'96 system for a number of cases. In the base case, $C o S$ was found to be approximately twice as high as $C o U$. Sensitivities with regards to the load level and the level of uncertainty in the system show that this is not a general result, but that the cost depends strongly on the network configuration.

\section{ACKNOWLEDGMENT}

This research work described in this paper has been carried out within the scope of the project "Innovative tools for future coordinated and stable operation of the pan-European electricity transmission system (UMBRELLA)", supported under the 7th Framework Programme of the European Union, grant agreement 282775 .

\section{REFERENCES}

[1] Liu, Z. et al., "Challenges, experiences and possible solutions in transmission system operation with large wind integration," in 11th International Workshop on Large-Scale Integration of Wind Power into Power Systems, Lisbon, Portugal, 2012.

[2] D. S. Kirschen and D. Jayaweera, "Comparison of risk-based and deterministic security assessments," IET Gener. Transm. Distrib., vol. 1, no. 4, pp. 527-533, 2007.

[3] B. Stott and O. Alsaç, "Optimal power flow - a brief anatomy," in XII SEPOPE, Rio de Janeiro, Brasil, 2012.

[4] R. Christie, B. F. Wollenberg, and I. Wangensteen, "Transmission management in the deregulated environment," Proceedings of the IEEE, vol. 88 , no. 2, pp. 170-195, Feb. 2000.

[5] B. Borkowska, "Probabilistic load flow," IEEE Trans. Power App. Syst., vol. 88, no. 2, pp. 170-195, Feb. 2000.

[6] J. Usaola, "Probabilistic load flow in systems with wind generation," IET Gener. Transm. Distrib., vol. 3, pp. 1031-1041, 2009.

[7] Z. Hu and X. Wang, "A probabilistic load flow method considering branch outages," IEEE Trans. Power Syst., vol. 21, pp. 507-514, 2006.

[8] G. L. Viviani and G. T. Heydt, "Stochastic optimal energy dispatch," IEEE Trans. Power App. Syst., vol. PAS-100, pp. 3221-3228, July 1981.

[9] M. Vrakopoulou, K. Margellos, J. Lygeros, and G. Andersson, "Probabilistic guarantees for the N-1 security of systems with wind power generation," in PMAPS 2012, Istanbul, Turkey, 2012.

[10] F. Bouffard and F. D. Galiana, "Stochastic security for operations planning with significant wind power generation," IEEE Trans. Power Syst., vol. 23, pp. 306-316, May 2008.

[11] U. Focken, M. Lange, K. Mönnich, H.-P. Waldl, H. G. Beyer, and A. Luig, "Short-term prediction of the aggregated power output of wind farms - a statistical analysis of the reduction of the prediction error by spatial smoothing effects," J. Wind Eng. Ind. Aerodynam., vol. 90, pp. 213-246, Mar. 2002.

[12] S. Chatzivasileiadis, T. Krause, and G. Andersson, "Flexible AC Transmission Systems (FACTS) and Power System Security - A Valuation Framework," in IEEE PES GM, Detroit, 2011.

[13] S. Boyd and L. Vandenberghe, Convex Optimization. Cambridge, UK: Cambridge University Press, 2004.

[14] Reliability Test System Task Force of the Application of Probability Methods Subcommittee, "The IEEE Reliability Test System-1996," IEEE Transactions on Power Systems, vol. 14, no. 3, pp. 1010 -1020, Aug. 1999.

[15] F. Milano, C. Canizares, and A. Conejo, "Sensitivity-based securityconstrained opf market clearing model," in PSCE 06, 2006, pp. 418 -427 . 